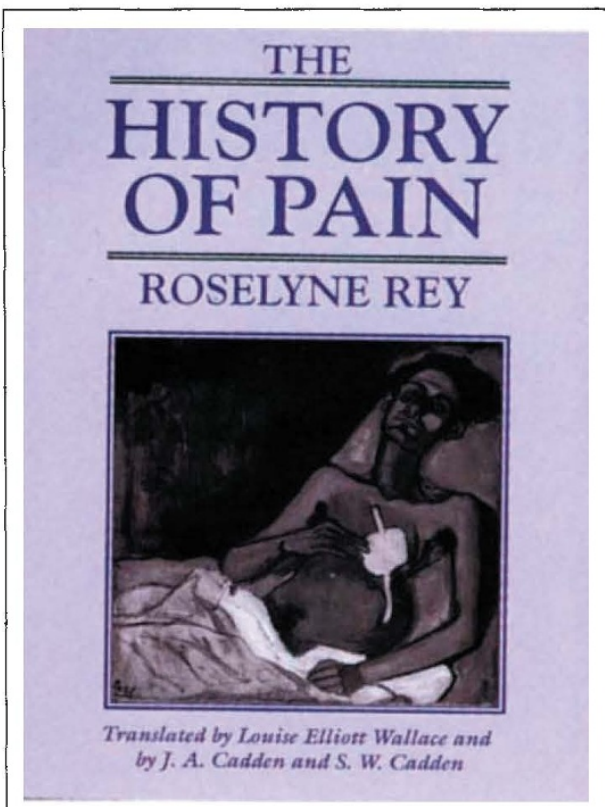

\section{The History of Pain}

\section{by Roselyne Rey, translated by Louise Elliott Wallace and J.A. \& S.W. Cadden Harvard University Press \\ Cambridge, Massachusetts, $\$ 39.95$ ISBN: 0-674-39967-6}

\section{REVIEWED BY GERT H. BRIEGER \\ Department of the History of Science, Medicine and Technology}

The Johns Hopkins University School of Medicine Baltimore, Maryland 21205

With the possible exception of death itself, no medical condition has been of greater concern to physicians and to their patients throughout history than has pain. Yet as recently as 1980 , the editors of a book on the meaning and management of pain noted the irony that the symptom that most commonly brings patients to their physicians was also one of the least understood. Similarly, we know quite a bit about the development of certain pain relievers and have often heard the story of anaesthesia, both general and local, but we know much less of the broader cultural history of pain. This historical gap is addressed by this splendid book.

Dr Roselyne Rey, a historian of medicine and the life sciences working in Paris, has dedicated her book "to discovering the ways in which physicians, physiologists, and neurologists have throughout the ages attempted to understand the practical mechanisms of pain and to find appropriate remedies for it." Her emphasis is on clarifying the changing scientific theories about the mechanisms of pain and the physiology of sensation.

Rey begins with the ancient writings of Homer and Hippocrates, but the heart of her book is devoted to the late eighteenth, nineteenth, and early twentieth centuries, and she is seemingly much more at home with European writers than she is with those on this side of the Atlantic. In the eighteenth century, as European society became increasingly secular, science became more and more separated from metaphysics, and scientists began to study the concept of sensibility.

The Enlightenment classification of pain is still useful today. Pain was described as four main types: tensive pain, that is manifested by the feeling of distension; gravitative, where there is a sensation of weight as in the accumulation of fluid in a body cavity, such as in ascites; pulsatory, as in inflammations; and pungitive pain, accompanied by a sensation of a sharp point or a stabbing. The feeling of pins and needles and the intense itching or pruritus are also included in the pungitive category of pain.

Pain has been easier to classify than to articulate in words. Virginia Woolf, in writing about an illness of her own in the early part of this century noted that, "The merest schoolgirl when she falls in love has Shakespeare or Keats to speak her mind for her, but let a sufferer to describe a pain in his head to a doctor and language at once runs dry."

The relief of pain has always been one of the prime tasks of doctors. How their patients have perceived pain and reacted to it has varied from culture to culture. Thus the story of pain, its mechanisms, as well as its toleration, lends itself well to a broad cultural history of medicine as Dr Rey has provided for us.

In the context of American medicine, Martin Pernick's A Calculus of Suffering (1985) used pain, the development of anaesthesia, and the striving of American medicine for increasing professionalism in nineteenth century America to address many of the same points. It is a failing of $\mathrm{Dr}$ Rey's book that she slides over the American parts of the story in a cursory and not very sure-footed way. John C. Warren, the Harvard surgeon in the successful use of ether in 1846, for instance, is described as Dr Warren of Cambridge University in Boston. Nor does she make use of Pernick's important book of a decade ago.
These and other minor lapses should not, however, detract from a solid scholarly accomplishment. Readers interested in the developments of neurology and neurophysiology on both sides of the Atlantic will be well rewarded. Whereas Rey discusses the debates over anaesthesia, which are well known and so occupy her but little, there is far more extensive description of the work of the physiologists such as Johannes Müller and the doctrine of specific nerve energies, the study of Wallerian degeneration, the work of Claude Bernard with curare, the Brown-Sequard studies of spinal cord sections and the clinical neurological studies of Weir Mitchell in this country and of Jean Charcot in France. While these and other clinical or physiological debates and studies were going on, the sociological aspects of pain were also being discussed. Do the rich and the poor feel pain in the same way? Is there a different perception of pain among different races?

Studies begun in the late nineteenth century on cerebral localization continued into the first decades of our own century. In England the neurologist Henry Head and the neurophysiologist Charles Sherrington brought the modern methods of experimentation and theory building to new understanding of the complexities of the nervous system. These developments have previously been described by historians, but it is the virtue of this book on pain to bring them together in one place.

This book is strong on the theory of medical practice as evidenced by good discussions of the evaluation of pain and what to ask patients. What is missing are the patients themselves, but the author did not intend to include them.

I am particularly aware of this aspect of the pain story because I have just read with medical students, A Whole New Life (1994) by Reynolds Price which is the report of his battle with a spinal cord tumour that rendered him paraplegic. But what Price really has written is an exquisite memoir of pain, its sensations and meanings, its life-interfering nature and its remarkable resistance to alleviation by his doctors.

Dr Rey has instead used pain as a key medical, biological, cultural and philosophical theme; she has fashioned a history of medicine around the concept of pain. It is thus a history of ideas more than of practices. She has undertaken a large task and carried it out with great success. To capture as much of our changing views of such a large topic as she has managed to do in a 350-page book is a considerable feat. 